\title{
LEOPOLDO CHIAPPO, A DIEZ AÑOS DE SU FALLECIMIENTO
}

\section{Leopoldo Chiappo, ten years after his death}

\author{
Ramón León* \\ Universidad Ricardo Palma
}

Perú

\section{Resumen}

La presente comunicación pasa revista al aporte conceptual de Leopoldo Chiappo (1924-2010) a la psicología peruana a diez años de su fallecimiento. Sus contribuciones en el campo de la neuropsicología y sus estudios en torno a la Divina Comedia, de Dante Alghieri, y el significado de esta obra para el saber psicológico son comentados, así como la importante labor docente formadora de psicólogos.

Palabras clave: Leopoldo Chiappo - Psicología.

\section{Abstract}

This communication reviews the conceptual contribution of Leopoldo Chiappo (1924-2010) to Peruvian psychology ten years after his death. His contributions in the field of neuropsychology and his studies around the Divine Comedy, by Dante Alghieri, and the significance of this work for psychological knowledge are discussed, as well as his important teaching work of training psychologists.

Keywords: Leopoldo Chiappo - Psychology.

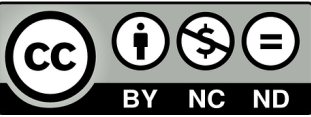

Cualquier uso que se haga de este artículo debe incluir: Autor / Título original de la publicación / ISSN.

* Docente de la Universidad Ricardo Palma. rld310850@yahoo.com.mx ORCID: 0000-0001-7380-6732 
La pandemia que asola a la humanidad, cobrando cientos de miles de vidas y sumiéndonos en una atmósfera de aislamiento, temor e incertidumbre, hace que vivamos concentrados en el día a día sin reparar en que cada año (este también) tiene sus efemérides, unas de recuerdo jubiloso y otras de rememoración melancólica. Estas líneas tienen el objetivo de conmemorar los diez años del fallecimiento de Leopoldo Chiappo Galli.

Leopoldo Chiappo Galli partió a la eternidad el 7 de marzo del 2010 dejando un gran vacío en la cultura nacional, pero también legando un importante patrimonio intelectual, que todavía espera un estudio detenido.

Educador, filósofo, literato, psicólogo, Chiappo fue autor de artículos, ensayos y libros que evidencian la vastedad de sus intereses, su erudición y la agudeza de su intelecto.

En los años del gobierno militar (1968-1980) participó activamente en las intensas discusiones en torno al desarrollo de un nuevo sistema educativo, más incluyente y democrático (Balarín 2019), cumpliendo un rol protagónico al lado de Augusto Salazar Bondy. Desde una nueva filosofía de la educación que hiciera justicia a las necesidades de la compleja sociedad peruana pasando por aspectos de la didáctica, hasta llegar a esbozar al que debía ser el nuevo hombre peruano, Chiappo hizo aportes a la Comisión de la Reforma Educativa, de la cual era integrante (Rojas Huaynates, 2014), formuló planteamientos, escribió artículos en revistas y periódicos (Chiappo, 1977). En una época en la cual la educación de los adultos era casi una novedad, reflexionó acerca de ella (Chiappo, 1975), y lo escrito por él en ese entonces nos lo muestra como un adelantado a lo que hoy es la andragogía.

Tanto antes como después de esos años, Chiappo dio a la luz trabajos en los que su interés por la filosofía y la literatura se expresó de modo sistemático. Su tesis de bachillerato, preparada cuando frisaba los 25 años, tuvo por título Posibilidades filosóficas de una interpretación de la experiencia mística (Chiappo, 1949), y su disertación doctoral abordó un tema en las antípodas: Consideraciones sobre la clase nula. Estudio de lógica matemática (Chiappo, 1951). Una y otra nos ofrecen un retrato de sus preocupaciones intelectuales en esos años de juventud, al explorar ese fascinante pero inconmensurable mundo que es el de la mística (tema que por cierto ha atraído a muchos), y al adentrarse en el intrincado dominio de la lógica matemática.

En las postrimerías de los años 70, en plena madurez intelectual, retornaría a la filosofía vinculándola con las demandas sociales de esos tiempos, a través de un ensayo sobre la figura paradigmática de Friedrich Nietzsche y lo que sus ideas significaban en el contexto político del momento (Chiappo, 1978).

En la literatura, de otro lado, ya desde muy temprano manifestó su interés por los escritos del gran místico hispano San Juan de la Cruz. Pasaría mucho tiempo y absolvería muchas lecturas hasta que, en los torrentosos años 80, los años del "desborde popular" en nuestro país (Matos Mar, 1984), encontrara en la Divina Comedia la obra a cuyo estudio se dedicaría hasta el fin de sus días.

Pero Leopoldo Chiappo es también una de las figuras más destacadas de la psicología peruana del siglo XX. Ubicado en la vertiente comprensiva del saber psicológico (Alarcón, 2000), es considerado como uno de los grandes teóricos nacionales de la especialidad.

Eso era indudablemente, un teórico. Como todos los intelectuales peruanos del siglo $\mathrm{XX}$, Chiappo emprendió tras la finalización de sus años universitarios un intenso programa autodidáctico, complementado después con estancias de estudio en los Estados Unidos, España e Italia. Su conocimiento de las lenguas europeas básicas, sus lecturas de obras de Kurt Goldstein, Aldous Huxley, Honorio Delgado, Ernst Cassirer y tantos más, aportaron lo suyo a ampliar y estructurar su formación intelectual, que le permitía ver los problemas (no solo los psicológicos, por cierto) con una perspectiva interdisciplinaria sazonada por sus puntos de vista personales.

El término teórico, sin embargo, tiene entre nosotros un cierto elemento peyorativo, al considerárselo como sinónimo de lo libresco y de lo ajeno a la experiencia práctica, valorada como la piedra de toque del profesional. En el caso del protagonista 
de este trabajo debemos rescatar el sentido primigenio del vocablo, que incide en la posesión de una sólida formación doctrinaria, que permite ir a la práctica con la seguridad de los conocimientos que se poseen.

En los años iniciales de la psicología como especialidad autónoma en la Universidad de San Marcos, se le encargó a Chiappo la asignatura de Diagnóstico Psicológico. Los cultivadores de la psicología clínica (que era, por cierto, la subespecialidad a la que todos los estudiantes apuntaban en ese entonces) saben perfectamente lo que el diagnóstico psicológico supone: un estricto estudio de caso por medio de entrevista al consultante y a sus allegados, una historia clínica que ofrezca la más completa panorámica biográfica y perfile con precisión la problemática motivo de la consulta, la aplicación, corrección y acertada interpretación de los tests, inventarios y pruebas proyectivas y la posterior integración de todo el cúmulo de información (y hasta de intuiciones provenientes de la experiencia del diagnosticador) en un documento (el informe psicológico) que debe describir, explicar e integrar los contenidos de la personalidad con la estructura de esta, destacando no solo lo carencial o negativo sino también lo potencial y positivo, sin perder de vista por supuesto la irrepetible índole de cada persona.

Ninguna tarea sencilla, por cierto, la de llevar a buen puerto un diagnóstico psicológico. Ni hoy, en que se cuenta con el DSM-V y con numerosos y didácticos tratados de psicopatología más el apoyo de procedimientos computarizados, ni cuando Chiappo enseñaba esa asignatura. Y mucho menos entonces, en que -como sucede hasta hoy- una teoría propone marcos e indicadores interpretativos que la otra desecha o relativiza, y en que además ya se comenzaba a tener clara conciencia de que las presunciones diagnósticas son constructos socioculturales y no realidades independientes de que sean denominadas como tales (Bogenschutz \& Nurnberg 2000, Williams 2017). Es decir, que "las enfermedades o los trastornos son constructos que nos ayudan a comprender a los enfermos, y a manejarnos mejor con los problemas clínicos" (Echevarría Pérez, 2015, p. 114).

De otro lado, no era mucha en nuestro medio la disponibilidad de material bibliográfico: aparte de algunos tests y pruebas proyectivas (el de Matrices Progresivas de Raven, el WAIS y el Test de Apercepción Temática son mencionados por el propio Chiappo 1957b), como el Psicodiagnóstico de Rorschach dado a conocer entre nosotros en artículo publicado en la Revista de Psiquiatría y Disciplinas Conexas (Rorschach, 1924), y de Los tests, de Béla Székely (1947), una obra de consulta obligatoria por esos años, solo se podía encontrar el Manual de diagnóstico psicológico de Richard Meili (1953), valioso libro pero orientado más bien al terreno de las pruebas psicológicas. Podemos suponer que el voluminoso libro El hombre normal, ese otro desconocido, del psiquiatra argentino Alejandro Raitzin (1937), pudo también formar parte de la bibliografía empleada para la asignatura de Diagnóstico Psicológico, dado que en la parte teórica contenía una detenida discusión sobre la normalidad, en tanto que en un extenso apéndice daba a conocer numerosos procedimientos psicométricos para el estudio de la personalidad.

Chiappo, con su propia experiencia clínica, más las lecturas de obras como el Curso de psiquiatría (Delgado, 1953) y La personalidad y el carácter (Delgado, 1943), de Honorio Delgado, y libros en otros idiomas adquiridos durante sus estancias en el extranjero, pudo desarrollar este curso (Chiappo. 1958b).

También en esos años Chiappo cumplió un activo trabajo de investigación (con un cimiento teórico importante) de temas neuropsicológicos, cuando esta especialidad se encontraba en ciernes, como lo acreditan sus publicaciones (Chiappo, 1955, 1957b, 1958a, 1959a, 1959b). El entonces Hospital Obrero (hoy conocido como Hospital Almenara) contaba con un servicio de neurología, en el cual nuestro autor pudo llevar a cabo importantes y sistemáticas investigaciones en los pacientes, las mismas que extendió después a pacientes con trastornos esquizofrénicos en el Hospital Víctor Larco Herrera. Estas experiencias además le permitieron elaborar un procedimiento de evaluación al que denominó el test noético-perceptivo, al que nos referiremos más adelante.

No menos importante es su labor docente, cumplida en la casa sanmarquina y después en la Universidad Peruana Cayetano Heredia, que fue su 
centro fundamental de trabajo y de investigación. En 1975 se fundó la especialidad de psicología de esa casa de estudios y él fue docente de la misma hasta su retiro.

Ganado por su admiración hacia la obra de Dante Alighieri, Chiappo se convirtió con el paso de los años en un "dantólogo a tiempo completo". La frecuentación de la Divina Comedia le permitió no sólo profundizar en su conocimiento, sino también establecer elementos de la difícil realidad subjetiva de los peruanos en los años en los que el terrorismo y la grave crisis económica ponían a prueba a todo el país. Fue así como en 1983 publicó Dante y la psicología del infierno (Chiappo, 1983). El estilo elegante de la argumentación, la selección precisa de situaciones, contextos y personajes de la Divina Comedia, y la agudeza y pertinencia de las observaciones hicieron que esta obra fuera recibida con gran interés y tuviera amplia lectoría. No faltaron algunas críticas que llamaban la atención acerca de la "lejanía" de esa obra con respecto a la realidad sombría del Perú en esos años, reclamando de su autor la concentración en los temas que urgían (la desocupación, el avance del terrorismo, la informalidad, etc.). A esto solía responder Chiappo que la libertad académica permitía que el investigador y estudioso se dedicara a analizar los temas de su interés, más allá de la importancia de los grandes problemas sociales (a los cuales, como hemos señalado, se había abocado durante su participación en la Reforma Educativa de los años 70). En 1986 la Universidad Peruana Cayetano Heredia lanzó la segunda edición de Dante y la psicología del infierno.

Muchas son las obras literarias que han despertado el interés y han movido a su estudio a psiquiatras y a psicólogos. Freud dedicó un ensayo a los hermanos Karamazov (Freud 1968) y dos seguidores suyos, Marie Bonaparte (1949) y Otto Rank (1922), escribieron sendos estudios sobre Edgar Allan Poe, el misterioso escritor norteamericano, y sobre el personaje de Don Juan. Literatos, poetas, obras teatrales y novelas han sido reiteradamente estudiados por parte de psiquiatras y psicólogos desde diferentes ángulos (Kohut, 1957, Mitscherlich, 1982, Gissi, 2002, Dillon, 2009, Oyebode, 2009, 2012, Rosenzweig, 2009, Montesinos, 2014).

Vistos unos como personajes excéntricos (Balzac), otros como seres marcados por un destino trágico (Fedor Dostoievsky, Oscar Wilde) y algunos como visionarios (Aldous Huxley, George Orwell) y moralistas (Leon Tolstoy), los literatos han llamado siempre la atención del público en general y de los especialistas en psicología y en la psiquiatría, pues todos reconocen que rinden valiosos servicios útiles a la sociedad, el más importante de los cuales es "capacitarnos para vernos a nosotros mismos como otros nos ven" (Lessing, 2007, p. 21).

¿Qué fue lo que despertó el interés de Chiappo por la Divina Comedia, una de las grandes obras de la literatura universal pero, en efecto, proveniente de una cultura diferente a la nuestra y de un tiempo muy lejano? Él mismo lo explica en las líneas que transcribimos seguidamente:

Uno de los aspectos más fascinantes de la Comedia de Dante es su realismo descriptivo, la fuerza alucinatoria de las imágenes que las palabras despiertan. Esa es la excelencia de internarse, literalmente, adentrarse en el poema. Esa su fascinación. Se trata de visibilidad plástica de las escenas dantianas. Las palabras estructuradas en endecasílabos, suaves o fuertes, dulces o ásperos, como la música, son como ventanas por donde asomarnos a los escenarios vivos de intensa sensibilidad imaginal. Todo el enorme edificio de la Comedia con sus espacios, tiempos y eternidades, se sostiene sobre el solar sutil de sus tercetos encadenados. Y el poema, hecho de aire, del mismo material evanescente de las palabras, se ha fijado en pergaminos por obra paciente de laboriosos amanuenses, luego estampados, muchas veces y primorosamente por los impresores. Y en el libro, códice manuscrito o volumen impreso, podemos transformar, mediante la lectura silenciosa, el aire de la palabra en discurrir interno de palabras inmaterializadas que resuenan quedamente sin ruido y nos abren espacios perceptibles a la imaginación y a nuestra sensibilidad inteligente (Chiappo 1987, vol. 1: pp. 29-30).

Tenía razón Chiappo, pues

La imaginación de Dante es una imaginación visual. Es una imaginación visual en un sentido 
diferente de la de un pintor moderno de naturaleza muerta: es visual en el sentido de que vivió en una edad durante la cual los hombres todavía veían visiones. Era un hábito psicológico, cuyo arte hemos olvidado, pero tan bueno como cualquiera de los nuestros. Nosotros no tenemos nada más que sueños, y hemos olvidado que ver visiones -una costumbre ahora relegada a los extraviados y a los ignorantes- fue alguna vez un género de ensueño más significativo, interesante y disciplinado (Eliot, 1994, p. 301).

Rendido ante la grandeza del opus dantiano y concentrado de allí en adelante en su estudio, Chiappo sistematizó sus primeras ideas en el ya mencionado Dante y la psicología del infierno, pero no le bastó con eso, pues siguió ampliando sus lecturas de especialistas sobre el gran vate. Como ejemplo está su conocimiento de un amplio ensayo, conceptualmente audaz, El esoterismo de Dante, de René Guénon (1989), filósofo francés convertido al islamismo y autor de trabajos sobre metafísica, religión y ritos iniciáticos. Y así, años después, apareció una obra monumental en tres volúmenes, las Escenas de la Comedia (Chiappo, 1987-1990), en las que el acento está puesto en el análisis literario sin que falten consideraciones de importancia para la psicología y la filosofía.

Este resumen de su obra no puede concluir sin mencionar los trabajos que Leopoldo Chiappo dedicara a su maestro Honorio Delgado (Chiappo, 1994; véase además, entre otros, Chiappo, 1957a, 1992). Espíritu universal, con un gran interés por la ciencia psicológica evidenciado a través de su tesis doctoral en ciencias (Delgado, 1923) y de Psicología que escribiera al alimón con Mariano Iberico (Delgado \& Iberico, 1933), Delgado ha sido la figura más importante de la psiquiatría hispanohablante del siglo XX. Su vinculación con Freud y el psicoanálisis en los primeros años de su carrera, su permanente tarea difusora de los avances en materia de psiquiatría, psicología y filosofía europeas, su permanente producción intelectual, hacían de él una de las más destacadas figuras de la cultura nacional. Sus repetidas conferencias concitaban numeroso público, sus libros (especialmente Psicología y su Curso de psiquiatría) eran lecturas imprescindibles para todo aquel que se interesara por esas disciplinas; sus clases en la
Facultad de San Fernando eran seguidas con gran atención por parte de sus alumnos (Bustamante Ruíz, 1986, Mariátegui, 1993). Miró Quesada (1970: 26) nos ofrece un retrato de la atmósfera característica de sus clases:

Corría el año de 1928. Honorio Delgado, introductor del psicoanálisis en América Latina, médico, psiquiatra, psicólogo, filósofo, personalidad de fama internacional, había aceptado dictar el curso de Psicología en la Facultad de Letras de la Universidad Nacional Mayor de San Marcos. El día de la clase inaugural los estudiantes no cabían en el aula. La expectativa, el entusiasmo ante la palabra del maestro eran inmensos. Honorio Delgado comenzó su curso rodeado de la admiración de cuantos lo escuchaban.

En el grupo discipular que rodeaba a Delgado, conformado por médicos y en especial por psiquiatras, la excepción la constituía Chiappo, formado en las humanidades. Asistente a sus clases y lector de sus obras, sin embargo, siempre se consideró discípulo de Delgado. Quienes lo visitábamos en la universidad o en su domicilio solíamos encontrarlo releyendo alguna obra de su maestro, cuyos escritos son frecuentemente mencionados en los diferentes trabajos que publicó a lo largo de su vida. Un ejemplo lo tenemos en "Honorio Delgado y la imagen del nuevo hombre" (Chiappo, 1970: 14), artículo en el cual, si bien reconoce que su maestro "no ha desarrollado específica y explícitamente ningún trabajo sobre la imagen del nuevo hombre", intenta "construir la imagen del nuevo hombre y desarrollarla como el resultado de un diálogo fundamental con el pensamiento de un filósofo peruano [Delgado, N. del A.] en función de la concreta realidad actual".

A diez años de su desaparición física podemos preguntarnos qué perdura de las ideas y los trabajos de Leopoldo Chiappo, especialmente para la psicología.

Debemos anotar en primer lugar su gran interés por los temas neuropsicológicos, que probablemente habrían continuado en el centro de sus preocupaciones de no haber sido ganado por los temas educativos (que cobraron gran importancia en los años 70 en el Perú) y por el desempeño de cargos administrativos en la 
casa de estudios sanmarquina, cuya biblioteca central fue dirigida por Chiappo en 1959 (apartándose de esa universidad en 1961, en solidaridad con la renuncia masiva de los profesores de la Facultad de Medicina encabezados por Honorio Delgado y Alberto Hurtado).

Tras su renuncia a San Marcos, Chiappo formó parte del grupo de profesores fundadores de la que hoy es la Universidad Peruana Cayetano Heredia, pero que en ese momento asumió el nombre de Universidad Peruana de Ciencias Médicas y Biológicas. La incertidumbre y el trabajo duplicado con el fin de forjar una casa de estudios literalmente de la nada y solo en base al entusiasmo y compromiso de sus docentes cobró su tributo en términos de tiempo que de otro modo se habría podido dedicar a la investigación.

La lectura de los trabajos neuropsicológicos de Chiappo permite reconocer un enfoque interdisciplinario, que integra a la consideración de los aspectos neurológicos, la psicología, la psicopatología, la filosofía y la lingüística, como queda ejemplificado en la extensa comunicación en la que da a conocer la aplicación de la prueba noético-perceptiva en esquizofrénicos (Chiappo 1957b). En esto, sigue a su maestro, Honorio Delgado, como a Kurt Goldstein, uno de los grandes neurólogos de la época y precursor de las neurociencias.

La prueba noético-perceptiva es un reactivo psicológico de factura nacional. Se trata, para emplear las palabras del propio Chiappo, de "unas láminas curiosas e ingeniosas de pura fantasía fotográfica", de 17 x 13 cms., "que contienen figuras esquemáticas logradas mediante fotografías de hebras de lana y trozos de hilo fino, dispuestos, ingeniosamente, de manera tal que aparecen nítidamente como paisajes y escenas románticas cotidianas" (Chiappo, 1957b: 110111). No se entiende por qué su uso no se ha difundido en la práctica clínica o en el trabajo investigatorio. Yabar Vílchez (2019) ha demostrado recientemente que este reactivo posee propiedades psicométricas que la hacen pasible de ser utilizada en la práctica clínica.

No menos digno de relievarse es su acercamiento pionero en nuestro medio a los temas que hoy constituyen el centro de la psicología positiva. Chiappo no se habría considerado -suponemos- un militante de este enfoque psicológico, pero su obra, en especial la de las últimas décadas de su vida, revelan su preocupación por explorar los aspectos saludables, o promotores del desarrollo del ser humano. El extenso artículo "El animal profundo: perfil psicológico del hombre" (Chiappo, 1999, p. 77) aborda la problemática de la condición humana desde la filosofía, la biología, la psicología, la psiquiatría, la sociología y la lingüística llegando a la conclusión de que

El hombre "conduce" su organismo porque es persona que tiene fines, y fines superiores, sublimes. Es que gracias a la persona como sujeto de la vida psíquica es posible en el animal humano algo nuevo e inusitado en la escala animal: la autodeterminación y la libertad como proyecto intrínseco de autorrealización de la propia existencia. En esto está el quid de la plenitud o del fracaso de la existencia humana. Se trata de la realización o de la frustración de un nivel de existencia superior, el de los motivos sublimes.

Y tiempo después, su libro sobre la psicología del amor (Chiappo, 2002) trata de uno de los sentimientos más valorados en la cultura occidental.

Lejano estuvo pues Leopoldo Chiappo de ese prejuicio psicopatológico muy difundido entre los psicólogos. Una vez más, la influencia de las ideas de Honorio Delgado y de Kurt Goldstein, que acuñara el término de autorrealización, se deja sentir en su visión del ser humano.

Hay también lo que llamaremos un legado formativo de Chiappo, quien dedicó una gran parte de su vida a la labor docente, cumplida de modo permanente primero en San Marcos y después en la casa herediana, pero también en cursos de postgrado en otras instituciones, y extendida a ciclos de conferencias y charlas ocasionales.

En la vida docente es que se pone de relieve el maestro. La docencia, para decirlo en términos muy gruesos, está constituida fundamentalmente las clases. "Clases buenas", "clases malas", "clases regulares": esas son las rotundas calificaciones que los alumnos suelen dar a las horas lectivas que reciben en las aulas universitarias. También son calificativos 
que empleamos los profesores para nuestro propio desempeño en el aula o para el desempeño de nuestros colegas.

Pero una clase es algo mucho más complejo. En ella se cumplen rituales (días y horas de inicio y de finalización, prácticas, exposiciones y exámenes programados) en escenarios ad hoc (el aula virtual en estos días), se desarrollan programas de estudio $\mathrm{y}$, por supuesto, se confrontan ideas: en toda clase se contrastan (aunque sea indirectamente) hechos del pasado con la realidad de hoy y con lo que puede ocurrir en el futuro. Como lo señala Collins (2000) las clases, los debates, los seminarios, las discusiones, tienen como marco referencial textos e interpretaciones del pasado, ya sea para criticarlos o como fundamento para los planteamientos del profesor o de los participantes en el debate.

Quienes alguna vez (o varias) asistimos a clases o debates en los que Leopoldo Chiappo participaba recordamos la claridad de sus planteamientos, la erudición que se expresaba de manera sutil y elegante, y la energía que ponía en la argumentación, no con el ánimo de imponer sus ideas, sino de estimular la participación y la profundización en los temas tratados. Lo que escribe Wiesse Rebagliati (2010, p. 147) acerca de una exposición de Chiappo en torno a la Divina Comedia puede ampliarse sin duda a lo que eran sus clases:

No exagero si digo que la rica humanidad de Leopoldo Chiappo se manifestaba también en un solo momento: el momento en que, frecuentemente ante un auditorio masivo, recitaba y explicaba La divina comedia. En una especie de coreografía expresiva, sus manos, su rostro y, muy especialmente, su voz se volvían vehículos del texto de la Comedia o del comentario agudo, enterado e imaginativo que Leopoldo le prodigaba y con el que, a la vez, él se revelaba como persona total. Leopoldo Chiappo era, en esos momentos, simultánea o sucesivamente, texto y comentario, lector e intérprete.

Es de destacarse, por último, el gran interés que Leopoldo Chiappo tenía en buscar en la literatura estímulos, intuiciones y hasta descripciones detenidas de gran utilidad para los psicólogos. Ya su maestro, Honorio Delgado (1953, p. 33), señalaba lo valiosas que son

Las descripciones de los grandes literatos que sufren y estudian sus flaquezas y anormalidades: Dostoievski, Amiel, Rousseau, Grillparzer, Hebbel, Cellini, Leopardi, De Quincey, Baudelaire, Maupassant, Proust y tantos otros.

Una observación que muchos otros especialistas en el terreno de las enfermedades mentales han hecho en repetidas oportunidades. Solo algunos ejemplos: Alajouanine (1972) ha comparado los ataques epilépticos con los estados de éxtasis experimentados por San Juan de la Cruz y plasmados en sus poemas; Gonther \& Schlimme (2010) han editado un volumen en el que analizan aspectos psiquiátricos de la vida y obra de Hölderlin, en tanto que Anderson (1971) ha discutido las características de la enfermedad de Strindberg.

Tanto para Chiappo como para Delgado habría sido muy agradable saber cómo es que hoy las neurociencias encuentran muchos atisbos de sus hallazgos y de los problemas que están en el centro de la investigación, en algunas obras literarias, como sucede en el caso de Borges (Quian Quiroga, 2012), de escritores como Proust (Lehrer, 2010) y Jane Austen (Jones, 2017), entre otros.

En la psicología peruana Chiappo representa una línea de trabajos de naturaleza fundamentalmente cualitativa que intentan rescatar el saber psicológico presente en obras artísticas (fundamentalmente las literarias) y en la filosofía para integrarlo en la comprensión de la subjetividad y la conducta del ser humano (León, 2018). En las canteras de los estudiosos peruanos de la conducta probablemente constituye una excepción, pero en modo alguno está solo en el contexto internacional como lo demuestran los escritos de Gissi (2002) en Chile, Vallejo-Nágera (1980) en España, Peters (1982) en Alemania, Kristeva (1997) en Francia, Scull (2015) en Estados Unidos, y Sorabji (2006) en Inglaterra. Y en la lejanía del tiempo aparece también la figura fundamental del argentino José Ingenieros (2011) en la misma línea de los autores antes mencionados. En todos ellos, así como en Freud 
y en Honorio Delgado, se trata de canalizar en los marcos de la ciencia psicológica el "magma bullente de lo anímico" (Casals, 2015) presente en las obras literarias.

La obra de Leopoldo Chiappo, como señalamos al comienzo de esta comunicación, espera todavía un estudio detenido. Literatos, educadores, filósofos y, por supuesto, también los psicólogos están convocados a dicha tarea analizando su opus desde sus respectivas especialidades.

\section{REFERENCIAS}

Alajouanine, T. (1972). Littérature et épilepsie L'expression littéraire de l'extase dans les romans de Dostoievski et dans les poems de Saint Jean de la Croix. En: Catteau, J., ed. Dostoievski, Cahiers de l'Herne, nro. 24, 309-324

Alarcón, R. (2000). Historia de la psicología en el Perú. De la Colonia a la República.Universidad Ricardo Palma.

Anderson, E. W. (1971). Strindberg's illness. Psychological Medicine, 1 (2), 104-117.

Balarín, M. (2019). ¿De qué hablamos cuando hablamos de la reforma educativa? Continuidades, discontinuidades y ausencias en los intentos recientes de reformar la educación básica en el Perú. En: Portocarrero, F. \& Vergara, A., eds. Aproximaciones al Perú de hoy desde las ciencias sociales. Lima, Universidad del Pacífico, 263285.

Bogenschutz, M. P. \& Nurnberg, H. G. (2000). Classification of mental disorders. En: Sadock, B. J. \& Sadock, V. A., eds., Kaplan and Sadock's Comprehensive textbook of psychiatry (vol. 2). Lippincott Williams \& Wilkins Publishers, 824839.

Bonaparte, M. (1949). The life and works of Edgar Allan Poe. Imago.

Bustamante Ruíz, C. (1986). Imagen primera y postrera de cuatro médicos humanistas. Revista de Neuro-psiquiatría, 46, 28-34.
Casals, J. (2015). Afinidades vienesas. Sujeto, lenguaje, arte. Anagrama.

Chiappo, L. H. (1949). Posibilidades filosóficas de una interpretación de la experiencia mística. Lima: Universidad Nacional Mayor de San Marcos, tesis de bachillerato.

Chiappo, L. H. (1951). Consideraciones sobre la clase nula. Estudio de lógica matemática. Lima: Universidad Nacional Mayor de San Marcos, tesis de doctorado.

Chiappo, L. (1955). Investigación experimental acerca del proceso configurativo Prueba de Configuración noético-perceptiva en los afásicos. Revista de Neuro-Psiquiatria, 18 (3), 304-342.

Chiappo, L. H. (1957a). La psicología de Honorio Delgado. En: Homenaje a Honorio Delgado en su 65. aniversario, Tipografía Peruana, 82-106.

Chiappo, L. H. (1957b). Prueba de integración noético perceptiva en esquizofrénicos crónicos. Anales de la Facultad de Medicina (Lima, Universidad Nacional Mayor de San Marcos), 40 (1), 108-129.

Chiappo, L. H. (1958a). La evolución verbal-categorial en las lesiones cerebrales. Revista de NeuroPsiquiatría, 21 (3), 456-481

Chiappo, L. H. (1958b). Métodos de la psicología clínica. Revista del Viernes Médico (Lima), 9, 48- 56 .

Chiappo, L. H. (1959a). La alteración de la actitud abstracta y la prueba de configuración noéticoperceptiva en los pacientes con lesiones cerebrales. Revista de Neuro-psiquiatría, 22 (4), 693-710.

Chiappo, L. H. (1959b). The noetic-perceptive configuration test and impairment of the abstract attitude in brain-injured patients. Journal of Individual Psychology, 15, 93-99.

Chiappo, L. H. (1970). Honorio Delgado y la imagen del nuevo hombre. En: Tercer Congreso Peruano de Neuro-Psiquiatría "Honorio Delgado". Anales, vol. II, Lima, P. L. Villanueva, 13- 21.

Chiappo, L. H. (1975). ¿Pueden los adultos seguir aprendiendo? La Prensa (Lima), ediciones del 29 y 30 de mayo, y 4 de junio. 
Chiappo, L. (1977). Reforma Educativa Peruana: necesidad y esperanza. Nueva Sociedad, 33 (noviembre-diciembre), 49-64.

Chiappo, L. (1978). Nietzsche, dominación y liberación. Industrialgraf.

Chiappo, L. H. (1983). Dante y la psicología del infierno. Compañía de Seguros Atlas.

Chiappo, L. (1987-1990). Escenas de la Comedia. Estudios Dantianos. Lima: Concejo Nacional de Ciencia y Tecnología - Universidad Peruana Cayetano Heredia (vol. I, 1987, Estudios I-IV; vol. II, 1988, Estudios V-VIII; vol. III, 1990, Estudios IX-XII).

Chiappo, L. H. (1992). La concepción del hombre en Honorio Delgado. Apuntes (Lima, Universidad del Pacífico), 31, 55-62.

Chiappo, L. H. (1994). Presencia espiritual de Honorio Delgado. Universidad Peruana Cayetano Heredia.

Chiappo, L. (1999). El animal profundo: perfil psicológico del hombre. Persona (Lima, Universidad de Lima), 2, 37-78.

Chiappo, L. H. (2002). Psicología del amor. Peisa.

Collins, R. (2000). The sociology of philosophies. A global theory of intelectual change. Cambridge, Massachusetts - Londres: The Belknap Press of Harvard University Press.

Delgado, H. (1923). Rehumanización de la cultura científica por la psicología. Lima: Universidad Nacional Mayor de San Marcos, tesis para optar el grado de doctor en ciencias naturales.

Delgado, H. \& Iberico, M. (1933). Psicología.

Delgado, H. (1943). La personalidad y el carácter.

Delgado, H. (1953). Curso de psiquiatría.

Dillon, B. (2009). Nine tormented lives. Faber and Faber.

Echevarría Pérez, R. (2015). El diagnóstico psicopatológico y las clasificaciones de los trastornos mentales. En: Jarne, A. \& Talarn, A., comps., Manual de psicopatología clínica. Herder, 109-148.

Eliot, T. S. (1944). Los poetas metafísicos y otros ensayos sobre teatro y religión. Emecé.
Freud, S. (1968). Dostoievski y el parricidio. En: Obras completas, Biblioteca Nueva, vol. 2, 11361145

Gissi,J.(2002).Psicologíaeidentidadlatinoamericana. Sociopsicoanálisis de cinco Premios Nobel de Literatura. Universidad Católica de Chile.

Gonther, U. \& Schlimme, J. E., eds. (2010). Hölderlin und die Psychiatrie. Berlín: Psychiatrie Verlag.

Guénon, R. (1989). El esoterismo de Dante. Dédalo.

Ingenieros, J. (2011). El hombre mediocre. Capital Intelectual.

Jones, W. (2017). Jane on the brain: exploring the science of social intelligence with Jane Austen. Pegasus Books

Kohut, H. (1957). "Death in Venice" by Thomas Mann. A story about the disintegration of artistic sublimation. Psychoanalytic Quarterly, 26 (2), 206-228.

Kristeva, J. (1997). Sol negro, depresión y melancolía. Monte Ávila Editores Latinoamericana.

Lehrer, J. (2010). Proust y la neurociencia. Una visión única de ocho artistas fundamentales de la modernidad. Paidós.

León, R. (2018). La psicología. Pasado, presente y problemas. Universidad Ricardo Palma.

Lessing, D. (2007). Las cárceles elegidas. Fondo de Cultura Económica.

Mariátegui, J. (1993). Elogio de Honorio Delgado. Universidad Peruana Cayetano Heredia.

Matos Mar, J. (1984). Desborde popular y crisis del estado: el nuevo rostro del Perú en la década de 1980. Instituto de Estudios Peruanos.

Meili, R. (1953). Manual de diagnóstico psicológico, Morata.

Miró Quesada, F. (1970). Honorio Delgado, maestro. En: Tercer Congreso Peruano de NeuroPsiquiatría “Honorio Delgado”. Anales, vol. II, Lima, P. L. Villanueva, 26-28.

Mitscherlich, A. (1982). Psychopathographien des Alltags. Schriftsteller und Psychoanalyse. Frankfurt: Suhrkamp. 
Montesinos, T. (2014). Melancolía y suicidios literarios. De Aristóteles a Alejandra Pizarnik. Fórcola.

Oyebode, F. (2009). Mindreadings. Literature and psychiatry. Royal College of Psychiatrists.

Oyebode, F. (2012). Madness at the theatre. Londres: Royal College of Psychiatrists.

Peters, U. H. (1982). Hölderlin. Wider die These vom edlen Simulanten. Reinbek bei Hamburgo: Rowohlt

Quian Quiroga, R. (2012). Borges and memory. Encounters with the human brain. The MIT Press.

Raitzin, A. (1937). El hombre normal, ese otro desconocido. Joaquín Gil.

Rank, O. (1922). Die Don Juan Gestalt. Imago, 8, 142196.

Rojas Huaynates, J. (2014). Augusto Salazar Bondy: ideólogo y editorialista de la Reforma Educativa. En: Quiróz Ávila, R., ed., Actas del Congreso sobre Augusto Salazar Bondy, Lima, Universidad Nacional Mayor de San Marcos, 87-100.

Rorschach, H. (1924). Valor del experimento de interpretación de formas para la psicoanálisis. Revista de Psiquiatría y Disciplinas Conexas, 5 (4), 293-338.
Rosenzweig, M. (2009). El teatro de la enfermedad. Biblos.

Scull, A. (2015). Madness in civilization. A cultural history of insanity from the Bible to Freud, from the Madhouse to Modern Medicine. Princeton University Press.

Sorabji. R. (2006). Self. Ancient and modern insights about individuality, life, and death. The University of Chicago Press.

Székely, B. (1947). Los tests. Kapelusz.

Vallejo-Nágera, J. A. (1980). Locos egregios. Argos Vergara.

Wiesse Rebagliati, J. (2010). Leopoldo Chiappo, lector de Dante (1924-2010). Apuntes (Lima, Universidad del Pacífico), 66, 147-149.

Williams, R. (2017). La política del modernismo. Godot.

Yábar Vílchez, E. (2019). Fundamentos teóricos de la prueba de configuración noético-perceptiva de Leopoldo H. Chiappo G. y su utilidad práctica. Lima: Universidad Nacional Mayor de San Marcos, tesis para optar el grado de Magister en Psicología con mención en Psicología Clínica y de la Salud.

Fecha de recepción: 5 de noviembre 2020

Fecha de aceptación: 28 de noviembre 2020 